\title{
Accurate Solutions of Stress Intensity Factors of Standard Fracture Test Specimens
}

\author{
Akihide Saimoto $^{1, a}$, Fumitaka Motomura ${ }^{1, b}$ and Hironobu Nisitani ${ }^{2}$ \\ ${ }^{1}$ Department of Mechanical Systems Engineering, Nagasaki University, 1-14 Bunkyo-machi, \\ Nagasaki 8528521 Japan \\ ${ }^{2}$ Professor Emeritus, Kyushu University and Kyushu Sangyo University, Japan \\ as-aki@nagasaki-u.ac.jp, ’motomura@nagasaki-u.ac.jp
}

Keywords: Stress Intensity Factor, Linear Elastic Fracture Mechanics, Standard Specimen, Body Force Method.

\begin{abstract}
Practically exact solutions of stress intensity factor for several two-dimensional standard specimens were calculated and shown in numeric tables. The solutions were confirmed to converge until 6 significant figures through a systematical computation of discretization analysis. The convergence analyses were carried out by using a general purpose program based on a body force method.
\end{abstract}

\section{Introduction}

Highly accurate solutions of Stress Intensity Factors (SIFs) of standard specimens are indispensable for the measurement of material properties such as fracture toughness, crack propagation resistance and fatigue strength. SIFs of standard fracture test specimens have been published worldwide so that they could be referred easily by researchers and engineers. Most solutions in handbooks are reliable and accurate, but unfortunately, it is still true that there exists an inadequate solution which violates the expected accuracy. In the present study, practically exact numerical solutions of SIFs converged until 6 significant figures are shown as numeric tables. These solutions are obtained by using a hand-made general purpose stress analysis code based on the Body Force Method (BFM).

\section{The Body Force Method}

BFM was originally proposed by H. Nisitani in 1967 as a boundary method for general elastic problems. In BFM, an elastic problem is transformed into a problem of an infinite domain with appropriately distributed body forces and body force doublets along an imaginary boundary denoted by $\Gamma$. Generally speaking, the imaginary boundary is composed of two kinds of boundaries $\Gamma_{s}$ and $\Gamma_{d} . \Gamma_{d}$ is a double imaginary boundary along which the body force doublets are distributed to imitate a presence of crack. While $\Gamma_{s}$ is a single imaginary boundary along which the body forces are distributed to imitate a presence of non-crack boundaries. Based on the principle of BFM, the stress components at an arbitrary point $P$ is expressed as,

$$
\sigma_{i j}(P)=\sigma_{i j}^{\infty}(P)+\int_{\Gamma s} \sigma_{i j}^{*}\left(P, Q_{k}\right) \rho_{k}(Q) d \Gamma(Q)+\int_{\Gamma d} \sigma_{i j}^{*}\left(P, Q_{k l}\right) \gamma_{k l}(Q) d \Gamma(Q) .
$$

In Eq.1, $P$ and $Q$ are the reference and the source points, respectively. $\sigma_{i j}(P)$ is a component of stress, $\sigma_{i j}^{\infty}(P)$ is a component of initial stress, $\sigma_{i j}^{*}\left(P, Q_{k}\right)$ is a component of stress at $P$ due to a unit magnitude of point force at $Q$ acting in the $k$-th direction in an infinite domain and $\sigma_{i j}^{*}\left(P, Q_{k l}\right)$ is a component of stress at $P$ due to a unit magnitude of point force doublets at $Q$ into the $k$ - and $l$-th directions in an infinite domain. $\rho_{k}(Q)$ and $\gamma_{k l}(Q)$ are the unknown density functions of body 
force and body force doublets per unit length of single and double imaginary boundaries, respectively. That is, in BFM, solution of any elasticity problem can be obtained by solving Eq.1. In order to determine the unknown density functions, boundary conditions have to be examined precisely. In a typical numerical analysis, the unknown density functions are replaced by an elementary function of piecewise continuous and the integral expression of Eq. 1 is replaced by a set of simultaneous equations that express the boundary conditions. There proposed kinds of techniques which are useful for the precise determination of unknown density functions, however, the detailed introduction of individual techniques are omitted due to limited page space of this monograph. The interested readers should consult references [1-3].

\section{Table of SIF for Typical Specimens}

In the following subsections, table of SIF solutions for 5 types of typical fracture test specimen are shown. In these tables, $N$ stands for a number of divisions of each straight imaginary boundary. That is, each imaginary boundary was divided into several segments of equal length and the density function of body force or body force doublet was assumed to have a linear variation in each segment. As the division number $N$ increases, the obtained SIF converges monotonically to practically exact values. The calculation was continued until at least 6 significant figures converged. Center Cracked Plate Tension (CCT). The center cracked plate tension specimen is a one most frequently documented in articles so that there exits several reference solutions. Among them, Isida's solution [4] seems most reliable ever published. The present analysis was carried out for $1 / 4$ part of the whole specimen due to symmetry of the problem.
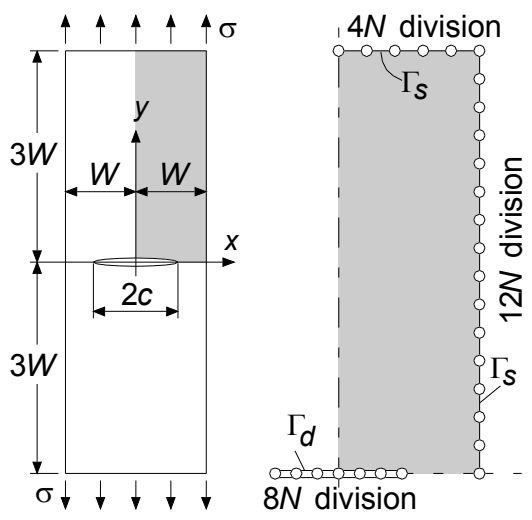

\begin{tabular}{|c|c|c|c|c|c|c|c|c|c|}
\hline \multirow{2}{*}{ div. } & \multicolumn{9}{|c|}{$c / W$} \\
\hline & 0.1 & 0.2 & 0.3 & 0.4 & 0.5 & 0.6 & 0.7 & 0.8 & 0.9 \\
\hline 1 & 1.00598 & 1.02448 & 1.05742 & 1.10862 & 1.18503 & 1.23000 & 1.48218 & 1.80584 & 2.55875 \\
\hline 2 & 1.00600 & 1.02459 & 1.05773 & 1.10932 & 1.18653 & 1.30299 & 1.48726 & 1.81232 & 2.57297 \\
\hline 5 & 1.00600 & 1.02459 & 1.05775 & 1.10938 & 1.18667 & 1.30333 & 1.48827 & 1.81609 & 2.57961 \\
\hline 10 & & 1.02459 & 1.05775 & 1.10938 & 1.18667 & 1.30333 & 1.48826 & 1.81606 & 2.57991 \\
\hline 20 & & & & & 1.18667 & 1.30333 & 1.48826 & 1.81602 & 2.57967 \\
\hline 40 & & & & & & & 1.48826 & 1.81601 & 2.57958 \\
\hline 60 & & & & & & & & 1.81601 & 2.57957 \\
\hline 80 & & & & & & & & 1.81601 & 2.57956 \\
\hline 100 & & & & & & & & & 2.57955 \\
\hline 120 & & & & & & & & & 2.57955 \\
\hline 160 & & & & & & & & & 2.57955 \\
\hline $\bar{\infty}$ & 1.00600 & 1.02459 & 1.05775 & 1.10938 & 1.18667 & 1.30333 & 1.48826 & 1.81601 & 2.57955 \\
\hline Ref.[4] & 1.0060 & 1.0246 & 1.0577 & 1.1094 & 1.1867 & 1.3033 & 1.4882 & 1.8160 & 2.5776 \\
\hline Ref.[5] & 1.0062 & 1.0254 & 1.0594 & 1.1118 & 1.1892 & 1.3043 & 1.4841 & - & - \\
\hline Ref.[6] & 1.0048 & 1.0208 & 1.0510 & 1.1000 & 1.1757 & 1.2921 & 1.4779 & 1.8075 & 2.5730 \\
\hline
\end{tabular}

Fig.1 Division model of CCT specimen and its non-dimensional SIF $\left(F_{I}=K_{I} / \sigma \sqrt{\pi c}\right)$

\section{Double Edge Cracked Plate Tension (DECT).}
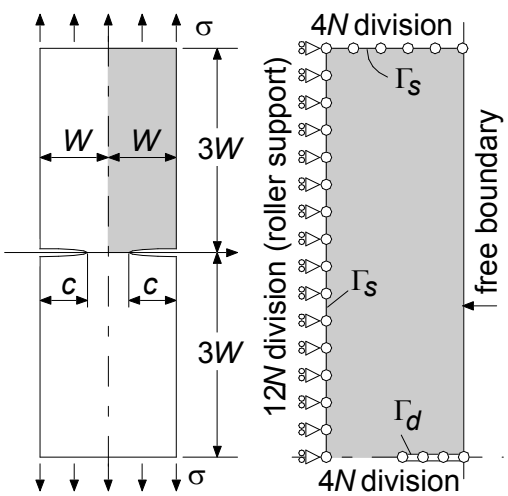

\begin{tabular}{|c|c|c|c|c|c|c|c|c|c|}
\hline \multirow{2}{*}{$\begin{array}{c}\text { div. } \\
\mathrm{N}\end{array}$} & \multicolumn{9}{|c|}{$c / W$} \\
\hline & 0.1 & 0.2 & 0.3 & 0.4 & 0.5 & 0.6 & 0.7 & 0.8 & 0.9 \\
\hline 1 & 1.11340 & 1.10867 & 1.11203 & 1.12929 & 1.16656 & 1.23382 & 1.35208 & 1.57592 & 2.10731 \\
\hline 2 & 1.11627 & 1.11125 & 1.11445 & 1.13159 & 1.16864 & 1.23528 & 1.35174 & 1.57185 & 2.11305 \\
\hline 5 & 1.11679 & 1.11173 & 1.11492 & 1.13207 & 1.16920 & 1.23601 & 1.35285 & 1.57346 & 2.11485 \\
\hline 10 & 1.11684 & 1.11177 & 1.11496 & 1.13212 & 1.16924 & 1.23606 & 1.35293 & 1.57366 & 2.11594 \\
\hline 20 & 1.11685 & 1.11178 & 1.11497 & 1.13212 & 1.16925 & 1.23607 & 1.35294 & 1.57369 & 2.11617 \\
\hline 40 & 1.11685 & 1.11178 & 1.11497 & 1.13212 & 1.16925 & 1.23608 & 1.35295 & 1.57370 & 2.11622 \\
\hline 60 & 1.11685 & 1.11178 & 1.11497 & 1.13212 & 1.16925 & 1.23608 & 1.35295 & 1.57370 & 2.11623 \\
\hline 80 & & & & & & & & & 2.11623 \\
\hline 100 & & & & & & & & & 2.11623 \\
\hline$\infty$ & 1.11685 & 1.11178 & 1.11497 & 1.13212 & 1.16925 & 1.23608 & 1.35295 & 1.57370 & 2.11623 \\
\hline Ref.[7] & - & 1.118 & 1.120 & 1.132 & 1.163 & 1.226 & 1.343 & 1.567 & - \\
\hline
\end{tabular}

Fig.2 Division model of DECT specimen and its non-dimensional SIF ( $F_{I}=K_{I} / \sigma \sqrt{\pi c}$ ) 
Single Edge Cracked Plate Tension (SECT).
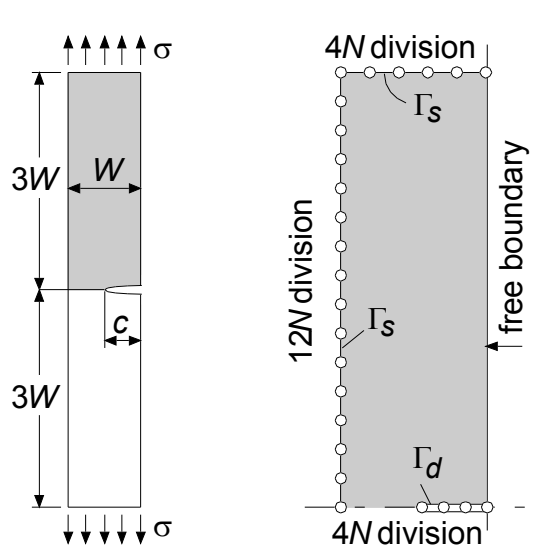

\begin{tabular}{|c|c|c|c|c|c|c|c|c|c|}
\hline \multirow{2}{*}{$\begin{array}{c}\text { div. } \\
\mathrm{N}\end{array}$} & \multicolumn{9}{|c|}{$c / W$} \\
\hline & 0.1 & 0.2 & 0.3 & 0.4 & 0.5 & 0.6 & 0.7 & 0.8 & 0.9 \\
\hline 1 & 1.18658 & 1.36352 & 1.65339 & 2.09907 & 2.79872 & 3.97479 & 6.31523 & 11.7964 & 37.0734 \\
\hline 2 & 1.18849 & 1.36642 & 1.65848 & 2.10895 & 2.81951 & 4.02065 & 6.35077 & 11.7831 & 34.3441 \\
\hline 5 & 1.18906 & 1.36718 & 1.65966 & 2.11107 & 2.82394 & 4.03170 & 6.35398 & 11.9355 & 34.3165 \\
\hline 10 & 1.18914 & 1.36729 & 1.65981 & 2.11132 & 2.82442 & 4.03278 & 6.35464 & 11.9516 & 34.5762 \\
\hline 20 & 1.18917 & 1.36731 & 1.65985 & 2.11139 & 2.82454 & 4.03302 & 6.35481 & 11.9544 & 34.6215 \\
\hline 60 & 1.18918 & 1.36732 & 1.65986 & 2.11141 & 2.82457 & 4.03310 & 6.35486 & 11.9552 & 34.6311 \\
\hline 100 & 1.18918 & 1.36733 & 1.65987 & 2.11141 & 2.82458 & 4.03311 & 6.35487 & 11.9553 & 34.6320 \\
\hline 120 & 1.18918 & 1.36733 & 1.65987 & 2.11141 & 2.82458 & 4.03311 & 6.35487 & 11.9553 & 34.6321 \\
\hline 140 & 1.18918 & 1.36733 & 1.65987 & 2.11141 & 2.82458 & 4.03311 & 6.35487 & 11.9553 & 34.6322 \\
\hline 160 & & & & 2.11141 & 2.82458 & 4.03311 & 6.35487 & 11.9553 & 34.6323 \\
\hline 180 & & & & & 2.82458 & 4.03311 & 6.35487 & 11.9553 & 34.6324 \\
\hline 200 & & & & & & & & 11.9553 & 34.6324 \\
\hline 220 & & & & & & & & 11.9553 & 34.6324 \\
\hline 240 & & & & & & & & & 34.6324 \\
\hline 260 & & & & & & & & & 34.6325 \\
\hline 280 & & & & & & & & & 34.6325 \\
\hline 300 & & & & & & & & & 34.6325 \\
\hline$\infty$ & 1.18918 & 1.36733 & 1.65987 & 2.11141 & 2.82458 & 4.03311 & 6.35487 & 11.9553 & 34.6325 \\
\hline Ref.[8] & - & 1.367 & 1.655 & 2.108 & 2.827 & 4.043 & 6.376 & 11.99 & - \\
\hline
\end{tabular}

Fig.3 Division model of SECT specimen and its non-dimensional SIF $\left(F_{I}=K_{I} / \sigma \sqrt{\pi c}\right)$

\section{Single Edge Cracked Pure Bending (SEB).}
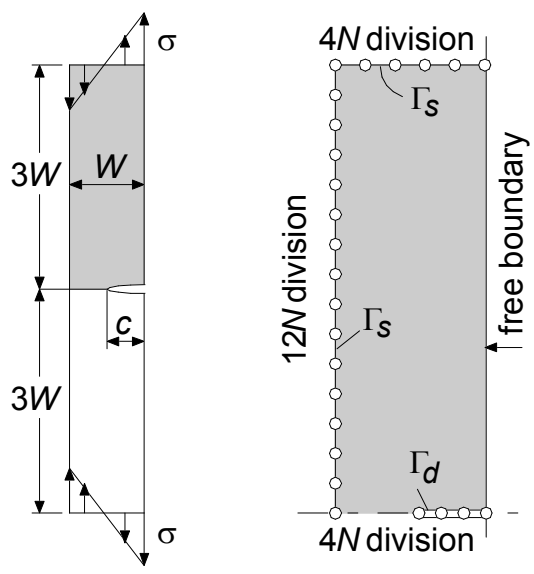

\begin{tabular}{|c|c|c|c|c|c|c|c|c|c|}
\hline \multirow{2}{*}{$\begin{array}{c}\text { div. } \\
\mathrm{N}\end{array}$} & \multicolumn{9}{|c|}{$c / W$} \\
\hline & 0.1 & 0.2 & 0.3 & 0.4 & 0.5 & 0.6 & 0.7 & 0.8 & 0.9 \\
\hline 1 & 1.04381 & 1.05105 & 1.11800 & 1.25068 & 1.47916 & 1.87837 & 2.65339 & 4.58093 & 13.2983 \\
\hline 2 & 1.04647 & 1.05443 & 1.12293 & 1.25875 & 1.49378 & 1.90668 & 2.70512 & 4.59964 & 12.3191 \\
\hline 5 & 1.04707 & 1.05516 & 1.12395 & 1.26038 & 1.49675 & 1.91314 & 2.72315 & 4.66770 & 12.3417 \\
\hline 10 & 1.04715 & 1.05525 & 1.12407 & 1.26057 & 1.49707 & 1.91377 & 2.72478 & 4.67473 & 12.4406 \\
\hline 20 & 1.04718 & 1.05528 & 1.12410 & 1.26061 & 1.49714 & 1.91391 & 2.72511 & 4.67600 & 12.4578 \\
\hline 40 & 1.04718 & 1.05528 & 1.12411 & 1.26063 & 1.49716 & 1.91395 & 2.72520 & 4.67629 & 12.4610 \\
\hline 60 & 1.04718 & 1.05528 & 1.12411 & 1.26063 & 1.49717 & 1.91396 & 2.72522 & 4.67635 & 12.4615 \\
\hline 80 & 1.04718 & 1.05528 & 1.12411 & 1.26063 & 1.49717 & 1.91396 & 2.72522 & 4.67637 & 12.4618 \\
\hline 100 & & & & & 1.49717 & 1.91396 & 2.72523 & 4.67638 & 12.4619 \\
\hline 120 & & & & & & 1.91397 & 2.72523 & 4.67639 & 12.4619 \\
\hline 140 & & & & & & 1.91397 & 2.72523 & 4.67639 & 12.4620 \\
\hline 160 & & & & & & & & & 12.4620 \\
\hline 180 & & & & & & & & & 12.4620 \\
\hline 200 & & & & & & & & & 12.4620 \\
\hline$\infty$ & 1.04718 & 1.05528 & 1.12411 & 1.26063 & 1.49717 & 1.91397 & 2.72523 & 4.67639 & 12.4620 \\
\hline Ref.[9] & - & 1.035 & 1.098 & 1.234 & 1.475 & 1.898 & 2.716 & 4.674 & - \\
\hline
\end{tabular}

Fig.4 Division model of SECT specimen and its non-dimensional SIF $\left(F_{I}=K_{I} / \sigma \sqrt{\pi c}\right)$

\section{Three Point Bending (3PB).}



\begin{tabular}{|c|c|c|c|c|c|c|c|c|c|}
\hline \multirow{2}{*}{$\begin{array}{c}\text { div. } \\
\mathrm{N}\end{array}$} & \multicolumn{9}{|c|}{$c / W$} \\
\hline & 0.1 & 0.2 & 0.3 & 0.4 & 0.5 & 0.6 & 0.7 & 0.8 & 0.9 \\
\hline 1 & 0.98805 & 0.98449 & 1.04675 & 1.17988 & 1.41451 & 1.82839 & 2.63002 & 4.57289 & 13.6491 \\
\hline 2 & 0.98220 & 0.97917 & 1.04146 & 1.17430 & 1.40869 & 1.82344 & 2.63071 & 4.56932 & 12.4163 \\
\hline 5 & 0.98050 & 0.97761 & 1.03987 & 1.17255 & 1.40657 & 1.82070 & 2.62706 & 4.56733 & 12.3269 \\
\hline 10 & 0.98027 & 0.97740 & 1.03966 & 1.17231 & 1.40629 & 1.82031 & 2.62639 & 4.56567 & 12.3209 \\
\hline 20 & 0.98022 & 0.97736 & 1.03961 & 1.17226 & 1.40623 & 1.82023 & 2.62625 & 4.56522 & 12.3168 \\
\hline 40 & 0.98021 & 0.97735 & 1.03961 & 1.17226 & 1.40622 & 1.82022 & 2.62622 & 4.56512 & 12.3156 \\
\hline 60 & 0.98021 & 0.97735 & 1.03960 & 1.17225 & 1.40622 & 1.82021 & 2.62622 & 4.56510 & 12.3153 \\
\hline 80 & 0.98021 & 0.97735 & 1.03960 & 1.17225 & 1.40622 & 1.82021 & 2.62622 & 4.56510 & 12.3153 \\
\hline 100 & & & & & & & & 4.56510 & 12.3152 \\
\hline 120 & & & & & & & & & 12.3152 \\
\hline 140 & & & & & & & & & 12.3152 \\
\hline 160 & & & & & & & & & 12.3152 \\
\hline$\infty$ & 0.98021 & 0.97735 & 1.03960 & 1.17225 & 1.40622 & 1.82021 & 2.62622 & 4.56510 & 12.3152 \\
\hline Ref.[10] & - & 0.988 & 1.045 & 1.179 & 1.416 & 1.831 & 2.630 & 4.543 & - \\
\hline
\end{tabular}

Fig.5 Division model of 3PB specimen and its non-dimensional SIF $\left(F_{I}=t W^{2} K_{I} / 3 P L \sqrt{\pi c}\right.$, $L=2 W, H=3 W$ ) 
In DECT (Fig.2), SECT (Fig.3), SEB (Fig.4) and 3PB (Fig.5) analyses, stress field due to a point force acting in a semi-infinite plate with free-of-traction edge was used as a fundamental solution $\sigma_{i j}^{*}\left(P, Q_{k}\right)$. Thus the boundary condition at an arbitrary point on the edge is satisfied automatically. When a crack emanates from a free edge, this fundamental solution improves the solution accuracy considerably.

\section{Conclusion}

Practically exact solutions of stress intensity factor of typical two-dimensional standard specimens are computed and presented. The shown solutions were confirmed to converge until 6 significant figures. These reliable solutions are useful for benchmarking, that is, the evaluation of numerical accuracy of the solutions obtained through other conventional numerical techniques such as finite element analysis. The versatile program base on the body force method for two-dimensional general elastic problems was found to be so powerful that the converged solutions were straightforwardly obtained simply by increasing the degree of boundary division.

\section{References}

[1] H. Nisitani, Journal of the Japan Society of Mechanical Engineers, Vol.70, No.580 (1967), p.627 (in Japanese)

[2] H. Nisitani, Mechanics of Fracture, 5, Sih, G. C. ed., Sijthoff \& Noordhoff, Chap. 1 (1978), p.1.

[3] H. Nisitani and A. Saimoto, Key Engineering Materials Vols. 251-252 (2003), p.97

[4] M. Isida, International Journal of Fracture, Vol.7, No.3 (1971), p.301

[5] R. E. Feddersen, ASTM STP-410 (1966), p.77

[6] W. T. Koiter, University of Technology; Report No. 314, Delft, Netherlands (1965)

[7] Y. Murakami (editor-in-chief) et. als., Stress Intensity Factor Handbook, Pergamon Press, Vol.1 (1987), p.6

[8] Page 9 of reference [7]

[9] Page 11 of reference [7]

[10] Page 13 of reference [7] 